\title{
F

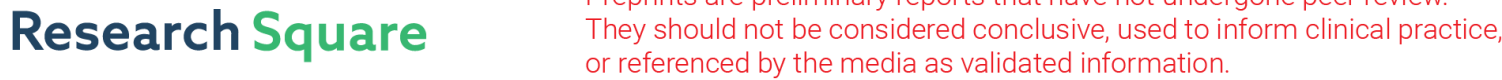 \\ Pediatric supratentorial glioblastoma: Experience at a Single Center in the past 6-year
}

\section{Yang Jiao ( $\nabla$ yangkkk123@126.com )}

Zhengzhou University First Affiliated Hospital https://orcid.org/0000-0002-3453-2887

\section{Meng Wang}

Zhengzhou University First Affiliated Hospital

\section{Xueyou Liu}

Zhengzhou University First Affiliated Hospital

\section{Junkuan Wang}

Zhengzhou University First Affiliated Hospital

\section{Zeming Wang}

Zhengzhou University First Affiliated Hospital

\section{Wenzheng Luo}

Zhengzhou University First Affiliated Hospital

\section{Yang Yu}

Zhengzhou University First Affiliated Hospital

\section{Keyan Xu}

Zhengzhou University First Affiliated Hospital

\section{Hongwei Sun}

Zhengzhou University First Affiliated Hospital

\section{Research Article}

Keywords: pediatric glioblastoma, supratentorial glioblastoma, imaging feature, long-term outcome, treatment

Posted Date: March 24th, 2021

DOI: https://doi.org/10.21203/rs.3.rs-333162/v1

License: (9) This work is licensed under a Creative Commons Attribution 4.0 International License. Read Full License 


\section{Abstract}

OBJECTIVE: Pediatric supratentorial glioblastoma (psGBM) is rare tumor of the central nervous system, the prognosis of it remains controversial due to their rarity. The aim of this study was to evaluate characteristics of pediatric supratentorial glioblastoma, effectiveness of modern treatment modalities, and detect factors related to prognosis

METHODS: Twenty-eight children under 18 years old were pathologically diagnosed with supratentorial glioblastoma at The First Affiliated Hospital of Zhengzhou University between 2014 and 2020.Epidemiological characteristics, clinical features, imaging features, functional outcomes, overall survival, progression-free survival according to multimodal treatments were reviewed retrospectively.

RESULTS: Median survival time of children with supratentorial glioblastoma was 11 months and median progression-free survival was 7 months. Half-, one-year and two-year overall survival probabilities were $64.3 \%, 42.9 \%$ and $14.3 \%$, respectively. Half-, one-year and two-year progression-free survival probabilities were $53.6 \%, 21.4 \%$ and $9.5 \%$, respectively. Extent of resection $(p=0.011)$ and postoperative concurrent chemoradiotherapy $(p=0.032)$ are independent factors of prognosis while gender, age, initial symptom, tumor side, enhancement character, preoperative KPS and with or without cystoid variation and necrosis are not. Three patients lived longer than 2 years, one patient was alive at 46 months after diagnosis and no evidence of recurrence was found.

CONCLUSION: Prognosis of pediatric supratentorial glioblastoma remains poor, and its prognosis may not be better than adult's. Preoperative KPS score is a risk factor, but isn't an independent risk factor. Concurrent chemoradiotherapy and gross total resection are the main methods to prolong life span.

\section{Highlights}

1. Pediatric supratentorial glioblastoma (psGBM) is rare tumor of the central nervous system

2. Complete resection of the psGBM with maximal safety remains the primary treatment to minimize the local progression.

3. Concurrent chemoradiotherapy does significantly prolong the overall survival and progression-free survival

4. Preoperative KPS score may predicts prognosis of patients.

5. the long-term clinical outcomes of psGBM after multimodal treatment remain

\section{Introduction}

Glioblastoma is one of the most malignant tumors in the central nervous system, which 5-year survival rate was less than 5\% [18], and it often happens to adults. Pediatric glioblastoma(pGBM) is not so common like adults, it constitutes around $3-7 \%$ of all children primary brain tumors[7,15,22]. In resent reports, there is many evidences found that pGBM may holds a different prognosis from adult part, and 
many indicators prove that pGBM is distinct from adult glioblastoma[5,15,22,25]. However, while the best therapy of pGBM is still on dispute, maximal surgical resection of tumor combined with concurrent chemoradiotherapy is considered to be the basic treatment which can provides a better prognosis and overall survival in many medical center. Due to the low incidence of pGBM, there is still a lack of large scale retrospective analysis of prognostic factors at present. In our study, we aim to analyzed the possible prognostic factors of pediatric supratentorial glioblastoma(psGBM) from the aspects of imaging features, treatment methods, tumor characteristics and effectiveness of modern therapy.

\section{Materials And Methods}

\section{Patient selection}

Twenty-eight consecutive patients were diagnosed with supratentorial glioblastoma at The First Affiliated Hospital of Zhengzhou University between 2014 and 2020खall patients were under 18 years old when firstly diagnosed. The clinical data of these patients were reviewed retrospectively after obtaining approval from the institutional review board of The First Affiliated Hospital of Zhengzhou University.

\section{Data collection}

The recorded information included patient age, sex, clinical manifestations, tumor side, tumor location, extent of resection (EOR), postoperative concurrent chemoradiotherapy (CCRT), preoperative KPS, enhancement character, cystoid variation and necrosis and relation with dura mater. We decided whether the relationship was close according to the location of the tumor and dura on MRI. The enhancement character was divided into annular enhancement and inhomogeneous enhancement (Typical pictures are shown in Fig.1). Long-term outcomes including progression free survival (PFS) and overall survival (OS). The period of overall survival was defined as the time interval between the initial treatment and the data of death or the last follow-up. The period of progression free survival was defined as the time between the initial treatment and the data of tumor recurrence based on imaging findings.

\section{Treatment and follow-up}

All patients received surgical treatment, the postoperative treatment including CCRT as per the Stupp protocol, chemotherapy only, radiotherapy only or untreated. Tumor tissue samples were collected during the operation for subsequent pathological examination. EOR were divided into total resection and subtotal resection. All patients were followed up by telephone consultation once every two months or outpatient review.

\section{Statistical analysis}

Age, PFS, and OS were analyzed as continuous variables. Age ( $>14$ or $<=14$ years), sex (male or female), tumor side (left or right), EOR (GTR or STR), postoperative CCRT (yes or no), preoperative KPS (>70 or $<=70$ ), enhancement character (annular enhancing or heterogeneous enhancing), cystoid variation and necrosis (yes or no). were analyzed as categorical variables. Kaplan-Meier method was used to estimate 
progression-free survival (PFS) and overall survival (OS). Differences between survival curves were assessed using log-rank test. Univariate Cox proportional hazard analysis was used to evaluate variables as prognostic factors for PFS and OS. Multivariate analysis was completed and included age, sex, tumor side, EOR, postoperative CCRT, preoperative KPS ( $>70$ or $<=70$ ), enhancement character, cystoid variation and necrosis. Statistical significance was determined by ANOVA for continuous variables. The Pearson chi-square test was used for categorical variables. Statistical significance was defined as a $p$ value < 0.05. All statistical analyses were completed using IBM SPSS 22.0 software.

\section{Result}

A summary of clinical and demographic characteristics of 28 patients is presented in Table 1 . The median age of the patients was 14 years, range 3 to 18 . The sex ratio of male to female is about 1.5区 1.The median OS is 11 months and median FPS is 7 months. The most common first symptom was headache, followed by nausea and vomiting. In 13 cases (46\%), tumors were found in the left hemisphere, while 15 cases (54\%) were found in the right hemisphere. The most common tumor location

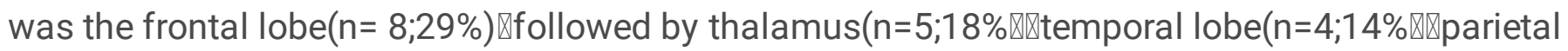
lobe( $n=3 ; 11 \% \mathbb{W}$ occipital lobe $(n=2 ; 7 \%$ 怄basal ganglia( $n=1 ; 4 \%)$. Five tumors $(18 \%)$ involved more than one lobe, included frontoparietal, frontotemporal, parietooccipital and temporo-occipital. Cystoid variation and necrosis were found in 24(85.7\%) patients. 16 cases were found to have a annular enhancement of tumor in MRI $\$ while the other 12 cases enhanced heterogeneously. We found a close relationship between the tumor and dura in 4 patients, however, none of them represent dura tail sign. After our evaluation, there is 10 cases that KPS $\leq 70 \otimes$ the other 18 cases have a KPS higher than 70 .

\section{Treatment and clinical outcomes}

In this group of 28 patients, fourteen patients (50\%) got gross total resection (GTR) and the rest of them got sub-total resection (STR). Fifteen(53.6\%) patients received concurrent chemoradiotherapy with temozolomide邓the dose of radiotherapy ranged from $54 \mathrm{~Gy}$ to $60 \mathrm{~Gy}$,four patients only received chemotherapy, nine patients got no treatment after operation. At the last follow-up, 25 died of tumor progression and 3 survived. Two patients underwent reoperation after recurrence and they got an overall survival of 24 months and 25 months respectively. There is a special recurrent case, the tumor showed multifocal growth when we firstly found it recurred after operation. Preoperative, postoperative and recurrent imaging data are shown in Fig. 2 The tumors of other recurrent cases recurred in situ or adjacent areas. The volume is larger and the course of disease is shorter than that of the first diagnosis.

\section{Kaplan-Meier survival analysis for study population}

The average follow-up time was 13 months (range 2 to 43 months). Kaplan-Meier plots of PFS and OS for 28 patients are presented in Fig. 3. Half-year, one-year and two-year survival rates were $64.3 \%, 42.9 \%$ and $14.3 \%$, respectively. The rate of progress-free was $53.6 \%$ in half a year, $21.4 \%$ in one year and $9.5 \%$ in two years. 


\section{Kaplan-Meier survival analysis based on prognostic factors}

Kaplan-Meier Survival Analysis identified the EOR $(p=0.008)$,postoperative CCRT $(p=0.009)$ and preoperative KPS $₫ p=0.017 \rrbracket$ were significant prognostic factors of OS(Fig4). It reveals that patients who receive GTR, postoperative CCRT and KPS>70 will have a better prognosis .EOR $(p=0.022)$ and postoperative CCRT) $(p=0.010)$ and preoperative KPS $₫ 0.040 \otimes$ were significant prognostic factors of PFS(Fig4). GTR, postoperative CCRT and preoperative KPS predicted better PFS. The half-year, one-year and two-year survival and progression free survival rates based on EOR and postoperative CCRT are shown in the Table 2. Age, sex, tumor side, enhancement character, cystoid variation and necrosis, were not prognostic factors for PFS and OSם

\section{Univariate and multivariate hazard ratio analysis of prognostic factors}

Univariate and multivariate results are presented in Table3.Univariate analysis identified GTR, postoperative CCRT and preoperative KPS were prognostic factors for better OS, with hazard ratios of $0.393,0.406$ and 0.476 , respectively. Univariate analysis identified GTR, postoperative CCRT and preoperative KPS were also prognostic factors for better PFS, with hazard ratios of $0.444,0.403$ and 0.476 , respectively. Multivariate analysis for prognostic factors was completed and included age, sex, tumor side, EOR, tumor grad, postoperative CCRT, preoperative KPS, enhancement character and cystoid variation and necrosis. Multivariate analysis identified EOR and postoperative CCRT were both prognostic factors for PFS and OS.

\section{Discussion}

The incidence of psGBM is very low, many related topics have been hotly discussed. We present a relatively large series of this disease from a single to explore further about its clinical, imaging features, long-term outcome, and prognostic factors. In our study, mean age of diagnosis is 12.8(14 in median). In previous studies, mean age of pediatric GBM patients ranges from 8.8 to 13.3 years, our result support the view that mean age at the time of diagnosis is around the beginning of the second decade of life $[5,15,22,25,29]$.In our series, male to female ratio was $3: 2$, this proportion is in line with previous studies[17], it is also in concordance with the majority of other studies reporting male predominance in both the pediatric and adult population $[7,15,20,22]$. We found no evidence of longer survival based on the patient's age or gender, which is comparable to data on pGBM available in literature $[5,20,32]$. The tumors were commonly located in the frontal, accounting for $29 \%$. Unfortunately, there are 10 people that their tumor involved multi-lobe or thalamus, this makes the operation more difficult and result in the low rate of gross total resection in this series. The primary symptoms of the patients were mainly related to increased intracranial pressure, about half of the patients had headache, nausea and vomiting, followed by limb dysfunction, and epilepsy, similar results can also be found in other literatures [17]. We believe that symptoms of intracranial hypertension may relate to the high degree of malignancy and rapid progress of the tumor. Epilepsy, as a symptom of cortical stimulation by tumor, usually occurs in patients 
with slow-growing brain tumors, such as oligodendroglioma. In general, the clinical manifestations of psGBM are generally nonspecific and depend on the tumor location and size.

Imaging findings in glioblastoma can often have prognostic significance as well, imaging differences between children and adults are still ambiguous. We divide our patients into two categories according to the enhancement characteristics: annular and inhomogeneous enhancement. In our study, the ratio of annular and inhomogeneous enhancement is 4:3(16:12). Median survival was 12 months and 9 months, respectively. It indicates psGBM with annular enhancement may have a better prognosis. Seung won Choi et al. have done in-depth research on the enhancement characteristic of glioblastoma, their study showed a better prognosis of annular enhancement [4]. GBM with annular enhancement implies a less heterogeneity in tumor. Genomic signatures associated with lysosomal activity and autophagy were enriched in inhomogeneous enhancement tumor, lysosome is considered as an important recycling organelle associated with autophagy, and also functions as a relay hub for signaling pathways driven by the mechanistic target of rapamycin complex 1 (mTORC1) [6,21]. Autophagy is known to have dual functions in respect to tumorigenesis [30], and its pro-tumorigenic role inducing the treatment resistance against temozolomide (TMZ) is often emphasized in GBM studies $[2,16,26]$. Nevertheless, in our study, enhancement character isn't a statistically significant prognostic factor, it may explain by the fact that our subjects are all children while their study population covers all ages and 144 patients were included. After all, many differences among patients of different age group have been found, and if possible, we should conduct a larger sample size study to evaluate the role of enhancement character in prognosis of psGBM.

Cystic necrosis is one of the common imaging features of GBM. We found it always appears in tumor with annular enhancement, however, some inhomogeneous enhanced tumor can also have cystic necrosis in central. Ji-ping Zhao et al. found that cystic necrosis happened in 7/8 adults diagnosed with epithelioid glioblastoma(eGBM) [33]. We found that children and adults had similar results囚in our study, such imaging changes were observed in $6 / 7$ of the children, however, we didn't find it a significant factor of prognosis, and there is less report about the relationship between cystic necrosis and prognosis .In general, cystic necrosis is considered to be a common feature of intracranial malignant tumors.

Glioblastoma originates from the white matter of the brain, and it usually occurs in the deep brain. However, it has been reported that glioblastoma can also be closely related to dura mater and even showed dura tail sign $[8,9,31]$, but pathological examination showed that the tumor only infiltrated the pia mater, no proliferative tumor cells were found in the adjacent dura. In our study, we found that there was a close relationship between dura mater and tumor in 4 patients (Fig.5). Relationship between the tumor and dura is so close that there is no obvious boundary on MRI, but none of them showed dura tail sign, and there was no dura attachment or thickening at surgery. Since this is a retrospective study, there is a lack of pathological evidence to find tumor cells in dura tissue. we didn't find any evidence to prove that close relationship between dura and tumor can affect prognosis, but the close relationship between the tumor and dura means that the tumor is located in the superficial part of the brain, which makes it easier to remove the tumor completely by surgery, this subject can be studied in the future. Studying the 
behavior of tumor is very helpful to the treatment of disease. All in all, the imaging research needs a further step.

As we all know, preoperative evaluation often plays an important role in clinical treatment, which determines the main way of treatment. Karnofsky Performance Status (KPS) score is a widely accepted method to evaluate the physical condition of patients. In our study, the mean preoperative KPS score was 71 (range 30-90). 17 (60.1\%) patients had KPS scores $\geq 70$, and their survival was better than the remaining patients on univariate analysis $₫ \mathrm{p}=0.017$ ). Many studies in adults have emphasized the prognostic significance of the preoperative performance status on survival [23,24]. We found it was not an independent risk factors on multivariate analysis, but in multivariate analysis, it can't be considered as a significant factor [5]. The possible reason is that the KPS value of the patients was influenced by many other prognostic factors, KPS value indirectly reflects the degree of preoperative disease development, meanwhile, KPS score is also a very important factor that determines the patient's ability to withstand the entire treatment. In our investigation, many parents give up active treatment for serious complications of radiotherapy and chemotherapy.

Maximal surgical resection followed by adjuvant radiotherapy with concomitant chemotherapy has become the current standard of care in glioblastoma [13,19, 20, 24,25,27]. Stupp et al. [28] demonstrated the efficacy of oral temozolomide in primary adult glioblastomas, chemotherapy has gradually become the mainstream treatment of glioblastoma in children. As for radiotherapy, its toxicity and long term side effects can never be ignored囚especially children are in an important stage of growth and development. In our center, resect as much as possible in a safe range followed by concurrent chemoradiotherapy is preferred. We found that postoperative CCRT is an effective way to prolong the survival time and delay the recurrence for psGBM patients. Although current studies have found many differences between childhood and adult GBM, but the effect of the same treatment in adults and children is not consistent, however, many new treatment options are still in active investigation $[3,11,14]$. Nowadays, electric field therapy is being hotly debated, it is an emerging treatment that can extend the life span of several months, it is also noninvasive and has little effect on children's growth and development. In the future, it may be an important way to prolong the life of psGBM patients.

Although we have collected patient data since 2014, the prognosis of psGBM has not been improved by modern treatment. In our study, the median OS was 11 months, and the half year survival rate was $64.3 \%$, one-year survival rate was $42.9 \%$, and two-year survival rate was $14.3 \%$.PFS of half-year, one-year and two-year are $53.6 \%, 21.4 \%$ and $9.5 \%$, respectively. Perkins et al. and Marina et al. reported median OS is 13.5 months in their study $[17,20]$. Karremann et al. performed a retrospective analysis on a relatively large group, nearly 200 children with GBM enrolled in their trials, the median OS was still about one year, just like in adults [12]. Some authors reported longer median OS time even reach 20 months and 43 months $[10,25]$. Deference of prognosis between children and adults is still a hot topic under discussion. We hold the belief that children have better prognosis and should be treated actively, however, our results show that there is no difference between the survival of our patients and that of adults. This may be attributed to the low gross-total resection rate in our group and some realistic factors, because of the high 
malignancy of this disease, many children didn't receive active treated. Studies have shown that gross total tumor resection can significantly delay the disease progression as well $[1,10,32,33]$. With the improvement of conventional microsurgical techniques and the application of advanced supplementary techniques such as diffusion tensor tractography (DTI), intraoperative MRI, and navigational guidance, the surgically related morbidity in critical locations has remarkably declined. Thus, we should take necessary measures to try our best to achieve gross total tumor resection.

At the last follow-up, three patients are still alive, one patient remained for 43 months without any evidence of recurrence, the other 2 patients accepted reoperation after the first recurrence and are in stable condition now. Although the current data is not enough for statistical analysis, it seems necessary to give active reoperation after recurrence. We will continue to follow up, looking for more related factors to prolong the survival period from the process of diagnosis and treatment.

\section{Conclusion}

Gross total resection combined with postoperative concurrent chemoradiotherapy is still a key factor for long-term survival. At the same time, preoperative functional status score also plays an important role, which indicates whether patients can survive the whole treatment process and obtain long-term survival.

\section{Abbreviation List}

psGBM: Pediatric Supratentorial Glioblastoma,

pGBM: Pediatric Glioblastoma,

eGBM: Epithelioid glioblastoma

CCRT: Concurrent Chemoradiotherapy,

GTR: Gross Total Resection,

STR: Subtotal Total Resection,

MRI: Magnetic Resonance Imaging,

OS: Overall Survival,

PFS: Progression-free Survival,

KPS: Karnofsky Performance Status

\section{Declarations}

Acknowledgements 
We thank all the patients who trusted us and all the physicians and staff who helped this study.

\section{Funding}

Not applicable

\section{Conflicts of interest}

Neither I nor my spouse/partner has a commercial interest, financial interest, and/or other relationship with manufacturers of pharmaceuticals, laboratory supplies, and/or medical devices or with commercial providers of medically related services.

\section{Availability of data and material}

The datasets used during the current study are available from the corresponding author on reasonable request.

\section{Code availability}

The datasets and code supporting the current study have not been deposited in a public repository but are available from the lead contact on request.

\section{Authors' contributions}

Not applicable

\section{Ethics approval}

The clinical data of these patients were reviewed retrospectively after obtaining approval from the institutional review board of The First Affiliated Hospital of Zhengzhou University.

\section{Consent to participate}

Not applicable

\section{Consent for publication}

Written informed consent for publication was obtained from all participants.

\section{References}

1. Adams H, Adams HH, Jackson C, Rincon-Torroella J, Jallo Gl, Quiñones-Hinojosa A.(2016) Evaluating extent of resection in pediatric glioblastoma: a multiple propensity score-adjusted population-based analysis. Child's nervous system : ChNS : official journal of the International Society for Pediatric Neurosurgery;32:493-503.https://doi.org/10.1007/s00381-015-3006-x. 
2. Buccarelli M, Marconi M, Pacioni S, De Pascalis I, D'Alessandris QG, Martini M, Ascione B, Malorni W, Larocca LM, Pallini R, Ricci-Vitiani L, Matarrese P.(2018) Inhibition of autophagy increases susceptibility of glioblastoma stem cells to temozolomide by igniting ferroptosis. Cell death \& disease;9:841.https://doi.org/10.1038/s41419-018-0864-7.

3. Cage TA, Mueller S, Haas-Kogan D, Gupta N.(2012) High-grade gliomas in children. Neurosurgery clinics of North America;23:515-23.https://doi.org/10.1016/j.nec.2012.04.007.

4. Choi SW, Cho HH, Koo H, Cho KR, Nenning KH, Langs G, Furtner J, Baumann B, Woehrer A, Cho HJ, Sa JK, Kong DS, Seol HJ, Lee JI, Nam DH, Park H.(2020) Multi-Habitat Radiomics Unravels Distinct Phenotypic Subtypes of Glioblastoma with Clinical and Genomic Significance. Cancers;12.https://doi.org/10.3390/cancers12071707

5. Das KK, Mehrotra A, Nair AP, Kumar S, Srivastava AK, Sahu RN, Kumar R.(2012) Pediatric glioblastoma: clinico-radiological profile and factors affecting the outcome. Child's nervous system : ChNS : official journal of the International Society for Pediatric Neurosurgery;28:205562.https://doi.org/10.1007/s00381-012-1890-x.

6. Degenhardt K, Mathew R, Beaudoin B, Bray K, Anderson D, Chen G, Mukherjee C, Shi Y, Gélinas C, Fan Y, Nelson DA, Jin S, White E.(2006) Autophagy promotes tumor cell survival and restricts necrosis, inflammation, and tumorigenesis. Cancer cell;10:51-64.https://doi.org/10.1016/j.ccr.2006.06.001.

7. Dolecek TA, Propp JM, Stroup NE, Kruchko C.(2012) CBTRUS statistical report: primary brain and central nervous system tumors diagnosed in the United States in 2005-2009. Neuro-oncology; 14 Suppl 5:v1-49.https://doi.org/10.1093/neuonc/nos218.

8. Guermazi A, Lafitte F, Miaux Y, Adem C, Bonneville JF, Chiras J.(2005) The dural tail sign--beyond meningioma. Clinical radiology;60:171-88.https://doi.org/10.1016/j.crad.2004.01.019.

9. Gupta S, Gupta RK, Banerjee D, Gujral RB.(1993) Problems with the "dural tail" sign. Neuroradiology;35:541-2.https://doi.org/10.1007/bf00588718.

10. Gupta S, Mallick S, Benson R, Haresh KP, Julka PK, Rath GK.(2017) Extent of surgical resection and adjuvant temozolomide improves survival in pediatric GBM: a single center experience. Child's nervous system : ChNS : official journal of the International Society for Pediatric Neurosurgery;33:951-6.https://doi.org/10.1007/s00381-017-3381-6.

11. Jung TY, Kim CY, Kim DS, Ra YS, Kim SH, Baek HJ, Choi HS, Kim IA.(2012) Prognosis of pediatric high-grade gliomas with temozolomide treatment: a retrospective, multicenter study. Child's nervous system : ChNS : official journal of the International Society for Pediatric Neurosurgery;28:10339.https://doi.org/10.1007/s00381-012-1786-9.

12. Karremann M, Butenhoff S, Rausche U, Pietsch T, Wolff JE, Kramm CM.(2009) Pediatric giant cell glioblastoma: New insights into a rare tumor entity. Neuro-oncology;11:3239.https://doi.org/10.1215/15228517-2008-099.

13. Klein R, Mölenkamp G, Sörensen N, Roggendorf W.(1998) Favorable outcome of giant cell glioblastoma in a child. Report of an 11-year survival period. Child's nervous system : ChNS : official 
journal of the International Society for Pediatric Neurosurgery;14:288-

91.https://doi.org/10.1007/s003810050228.

14. MacDonald TJ, Aguilera D, Kramm CM.(2011) Treatment of high-grade glioma in children and adolescents. Neuro-oncology;13:1049-58.https://doi.org/10.1093/neuonc/nor092.

15. Mahvash M, Hugo HH, Maslehaty H, Mehdorn HM, Stark AM.(2011) Glioblastoma multiforme in children: report of 13 cases and review of the literature. Pediatric neurology;45:17880.https://doi.org/10.1016/j.pediatrneurol.2011.05.004.

16. Munshi A.(2009) Chloroquine in glioblastoma-new horizons for an old drug. Cancer;115:23803.https://doi.org/10.1002/cncr.24288.

17. Nikitović M, Stanić D, Pekmezović T, Gazibara MS, Bokun J, Paripović L, Grujičić D, Sarić M, Mišković I.(2016) Pediatric glioblastoma: a single institution experience. Child's nervous system : ChNS : official journal of the International Society for Pediatric Neurosurgery;32:97103.https://doi.org/10.1007/s00381-015-2945-6.

18. Ostrom QT, Gittleman H, Liao P, Rouse C, Chen Y, Dowling J, Wolinsky Y, Kruchko C, Barnholtz-Sloan J.(2014) CBTRUS statistical report: primary brain and central nervous system tumors diagnosed in the United States in 2007-2011. Neuro-oncology;16 Suppl 4:iv1-

63.https://doi.org/10.1093/neuonc/nou223.

19. Ozbek N, Cakir S, Gursel B, Meydan D.(2004) Prognostic significance of seizure in patients with glioblastoma multiforme. Neurology India;52:76-8.

20. Perkins SM, Rubin JB, Leonard JR, Smyth MD, El Naqa I, Michalski JM, Simpson JR, Limbrick DL, Park TS, Mansur DB.(2011) Glioblastoma in children: a single-institution experience. International journal of radiation oncology, biology, physics;80:1117-

21.https://doi.org/10.1016/j.ijrobp.2010.03.013.

21. Rebecca VW, Nicastri MC, McLaughlin N, Fennelly C, McAfee Q, Ronghe A, Nofal M, Lim CY, Witze E, Chude Cl, Zhang G, Alicea GM, Piao S, Murugan S, Ojha R, Levi SM, Wei Z, Barber-Rotenberg JS, Murphy ME, Mills GB, Lu Y, Rabinowitz J, Marmorstein R, Liu Q, Liu S, Xu X, Herlyn M, Zoncu R, Brady DC, Speicher DW, Winkler JD, Amaravadi RK.(2017) A Unified Approach to Targeting the Lysosome's Degradative and Growth Signaling Roles. Cancer discovery;7:1266-83.https://doi.org/10.1158/21598290.Cd-17-0741.

22. Sánchez-Herrera F, Castro-Sierra E, Gordillo-Domínguez LF, Vaca-Ruiz MA, Santana-Montero B, Perezpeña-Diazconti M, González-Carranza V, Torres-García S, Chico-Ponce de León F.(2009) Glioblastoma multiforme in children: experience at Hospital Infantil de Mexico Federico Gomez. Child's nervous system : ChNS : official journal of the International Society for Pediatric Neurosurgery;25:551-7.https://doi.org/10.1007/s00381-008-0780-8.

23. Scott JN, Rewcastle NB, Brasher PM, Fulton D, MacKinnon JA, Hamilton M, Cairncross JG, Forsyth P. (1999) Which glioblastoma multiforme patient will become a long-term survivor? A population-based study. Annals of neurology;46:183-8. 
24. Shinojima N, Kochi M, Hamada J, Nakamura H, Yano S, Makino K, Tsuiki H, Tada K, Kuratsu J, Ishimaru Y, Ushio Y.(2004) The influence of sex and the presence of giant cells on postoperative longterm survival in adult patients with supratentorial glioblastoma multiforme. Journal of neurosurgery;101:219-26.https://doi.org/10.3171/jns.2004.101.2.0219.

25. Song KS, Phi JH, Cho BK, Wang KC, Lee JY, Kim DG, Kim IH, Ahn HS, Park SH, Kim SK.(2010) Longterm outcomes in children with glioblastoma. J Neurosurg Pediatr;6:1459.https://doi.org/10.3171/2010.5.Peds09558.

26. Sotelo J, Briceño E, López-González MA.(2006) Adding chloroquine to conventional treatment for glioblastoma multiforme: a randomized, double-blind, placebo-controlled trial. Annals of internal medicine;144:337-43.https://doi.org/10.7326/0003-4819-144-5-200603070-00008.

27. Sposto R, Ertel IJ, Jenkin RD, Boesel CP, Venes JL, Ortega JA, Evans AE, Wara W, Hammond D.(1989) The effectiveness of chemotherapy for treatment of high grade astrocytoma in children: results of a randomized trial. A report from the Childrens Cancer Study Group. Journal of neuro-oncology;7:16577.https://doi.org/10.1007/bf00165101.

28. Stupp R, Mason WP, van den Bent MJ, Weller M, Fisher B, Taphoorn MJ, Belanger K, Brandes AA, Marosi C, Bogdahn U, Curschmann J, Janzer RC, Ludwin SK, Gorlia T, Allgeier A, Lacombe D, Cairncross JG, Eisenhauer E, Mirimanoff RO.(2005) Radiotherapy plus concomitant and adjuvant temozolomide for glioblastoma. The New England journal of medicine;352:98796.https://doi.org/10.1056/NEJMoa043330.

29. Suri V, Das P, Pathak P, Jain A, Sharma MC, Borkar SA, Suri A, Gupta D, Sarkar C.(2009) Pediatric glioblastomas: a histopathological and molecular genetic study. Neuro-oncology;11:27480.https://doi.org/10.1215/15228517-2008-092.

30. White E.(2015) The role for autophagy in cancer. The Journal of clinical investigation;125:426.https://doi.org/10.1172/jci73941.

31. Wilms G, Lammens M, Marchal G, Demaerel P, Verplancke J, Van Calenbergh F, Goffin J, Plets C, Baert AL.(1991) Prominent dural enhancement adjacent to nonmeningiomatous malignant lesions on contrast-enhanced MR images. AJNR American journal of neuroradiology;12:761-4.

32. Yang T, Temkin N, Barber J, Geyer JR, Leary S, Browd S, Ojemann JG, Ellenbogen RG.(2013) Gross total resection correlates with long-term survival in pediatric patients with glioblastoma. World neurosurgery;79:537-44.https://doi.org/10.1016/j.wneu.2012.09.015.

33. Zhao JP, Cui CX, Wang JC, Su HW, Duan CF, Liu XJ.(2020) Multimodal MR Features of 8 Cases of Epithelioid Glioblastoma. BioMed research international;2020:9586806.https://doi.org/10.1155/2020/9586806.

\section{Tables}

Table 1 A summary of 28 patients characteristic. 


\begin{tabular}{|c|c|}
\hline characteristic & Value(\%) \\
\hline \multicolumn{2}{|l|}{ Age } \\
\hline Median & 14 \\
\hline Range & $3-18$ \\
\hline \multicolumn{2}{|l|}{ Sex } \\
\hline Male & $17(60.7)$ \\
\hline Female & 11(39.3) \\
\hline \multicolumn{2}{|l|}{ Initial symptom } \\
\hline Headache & $16(57.1)$ \\
\hline Nausea and vomiting & $14(50)$ \\
\hline Dyspraxia & $9(32.1)$ \\
\hline Seizure & $6(21.4)$ \\
\hline \multicolumn{2}{|l|}{ Tumor side } \\
\hline Left & $13(46.4)$ \\
\hline Right & 15(53.6) \\
\hline \multicolumn{2}{|l|}{ EOR } \\
\hline GTR & $14(50)$ \\
\hline STR & $14(50)$ \\
\hline \multicolumn{2}{|l|}{ Postoperative CCRT } \\
\hline Yes & 15(53.6) \\
\hline No & $13(46.4)$ \\
\hline \multicolumn{2}{|l|}{ Preoperative KPS } \\
\hline$\leq 70$ & 11(39.3) \\
\hline$>70$ & $17(60.7)$ \\
\hline \multicolumn{2}{|l|}{ Enhancement character } \\
\hline Annular enhancing & $16(57.1)$ \\
\hline Heterogeneous enhancing & $12(42.9)$ \\
\hline \multicolumn{2}{|l|}{ Relation of tumor and dura } \\
\hline Close & $4(14.3)$ \\
\hline
\end{tabular}

Page 13/20 


\begin{tabular}{|ll|}
\hline Not close & $24(85.7)$ \\
\hline Yes & \\
\hline No & $24(85.7)$ \\
\hline Tumor location & $4(14.3)$ \\
\hline Frontal & \\
\hline Parietal & $8(28.6)$ \\
\hline Temporal & $3(10.7)$ \\
\hline Occipital & $4(14.3)$ \\
\hline Thalamus & $2(7.1)$ \\
\hline Other & $5(17.9)$ \\
\hline
\end{tabular}

Table 2.Subgroup PFS and OS rates based on EOR and postoperative CCRT.

\begin{tabular}{|c|c|c|c|c|}
\hline \multirow[t]{2}{*}{ survival } & \multicolumn{2}{|l|}{ GTR } & \multicolumn{2}{|l|}{ STR } \\
\hline & PFS(\%) & OS(\%) & PFS(\%) & OS(\%) \\
\hline 6 months & 64.3 & 71.4 & 42.9 & 71.4 \\
\hline 1 year & 35.7 & 64.3 & 7.1 & 21.4 \\
\hline 2 years & 19 & 28.6 & 0 & 0 \\
\hline \multirow[t]{2}{*}{ survival } & \multicolumn{2}{|l|}{ CCRT } & \multicolumn{2}{|c|}{ Non-CCRT } \\
\hline & PFS(\%) & OS(\%) & PFS(\%) & OS(\%) \\
\hline 6 months & 64.3 & 71.4 & 42.9 & 71.4 \\
\hline 1 year & 35.7 & 64.3 & 7.1 & 21.4 \\
\hline 2 years & 19 & 28.6 & 0 & 0 \\
\hline
\end{tabular}

Table 3. Univariate and multivariate analysis of prognostic factors for PFS and OS 


\begin{tabular}{|c|c|c|}
\hline Prognostic & Univariate analysis & Multivariate analysis \\
\hline Factors & PFS OS & PFS OS \\
\hline \multicolumn{3}{|l|}{ Age } \\
\hline $\mathrm{HR}$ & 1.3301 .058 & 0.9980 .630 \\
\hline $95 \% \mathrm{Cl}$ & $\begin{array}{lll}0.601-2.950 & 0.480-2.332\end{array}$ & $0.396-2.515 \quad 0.254-1.568$ \\
\hline$P$ Value & 0.4610 .890 & 0.9970 .321 \\
\hline \multicolumn{3}{|l|}{ Sex } \\
\hline $\mathrm{HR}$ & 0.7490 .796 & 0.7740 .694 \\
\hline $95 \% \mathrm{Cl}$ & 0.333-1.684 $0.354-1.788$ & $0.292-2.052 \quad 0.247-1.954$ \\
\hline P Value & 0.4850 .796 & 0.6070 .489 \\
\hline \multicolumn{3}{|l|}{ Tumor side } \\
\hline $\mathrm{HR}$ & 0.9390 .880 & 0.7480 .443 \\
\hline $95 \% \mathrm{Cl}$ & $0.425-2.074 \quad 0.394-1.966$ & $0.262-2.141 \quad 0.146-1.348$ \\
\hline P Value & 0.8760 .756 & 0.5890 .152 \\
\hline \multicolumn{3}{|l|}{ EOR } \\
\hline $\mathrm{HR}$ & 0.4440 .393 & 0.3520 .267 \\
\hline $95 \% \mathrm{Cl}$ & $0.197-1.005 \quad 0.171-0.902$ & $0.141-0.881 \quad 0.096-0.741$ \\
\hline P Value & 0.0220 .008 & 0.0260 .011 \\
\hline \multicolumn{3}{|c|}{ Postoperative CCRT } \\
\hline $\mathrm{HR}$ & 0.4300 .406 & 0.3630 .299 \\
\hline $95 \% \mathrm{Cl}$ & $0.185-0.998 \quad 0.173-0.951$ & $0.151-0.868 \quad 0.100-0.900$ \\
\hline P Value & 0.0180 .014 & 0.0230 .032 \\
\hline \multicolumn{3}{|c|}{ Preoperative KPS } \\
\hline $\mathrm{HR}$ & 0.4760 .419 & 0.6070 .411 \\
\hline $95 \% \mathrm{Cl}$ & $0.203-1.116 \quad 0.175-1.004$ & $0.245-1.503 \quad 0.149-1.134$ \\
\hline P Value & 0.0390 .017 & 0.2800 .086 \\
\hline \multicolumn{3}{|c|}{ Enhancement character } \\
\hline $\mathrm{HR}$ & 0.7360 .810 & 0.7270 .830 \\
\hline $95 \% \mathrm{Cl}$ & $0.345-1.686 \quad 0.366-1.790$ & $0.308-1.715 \quad 0.331-2.079$ \\
\hline
\end{tabular}




\begin{tabular}{|c|c|c|}
\hline$P$ Value & $0.504 \quad 0.602$ & $0.467 \quad 0.691$ \\
\hline \multicolumn{3}{|c|}{ Cystoid variation and necrosis } \\
\hline HR & 0.7110 .708 & 0.6950 .384 \\
\hline $95 \% \mathrm{Cl}$ & $0.239-2.114 \quad 0.238-2.109$ & $0.223-2.162 \quad 0.104-1.416$ \\
\hline$P$ Value & $0.540 \quad 0.708$ & $0.695 \quad 0.151$ \\
\hline
\end{tabular}

PFS, progression-free survival; OS, overall survival; EOR, extent of resection; CCRT, concurrent chemoradiotherapy

\section{Figures}

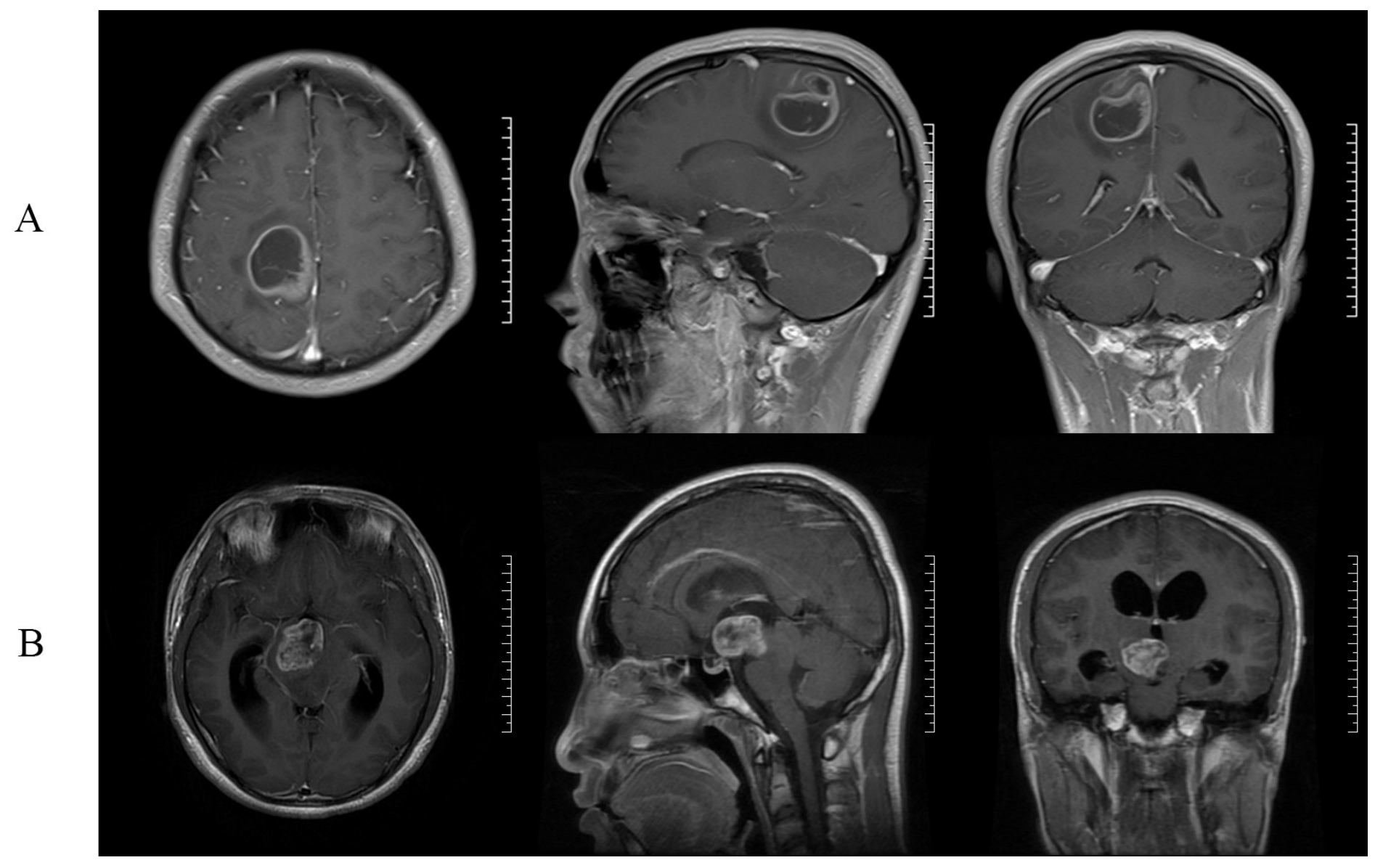

\section{Figure 1}

Typical magnetic resonance imaging (MRI) of annular enhancement and inhomogeneous enhancement. (A) Annular enhancement is characterized by obvious surrounding annular hyperintense signal and central homogeneous hypointense signal. (B) Inhomogeneous enhancement is characterized by inhomogeneous hyperintense signal and there was no significant correlation between hyperintense and hypointense signal areas. 
A

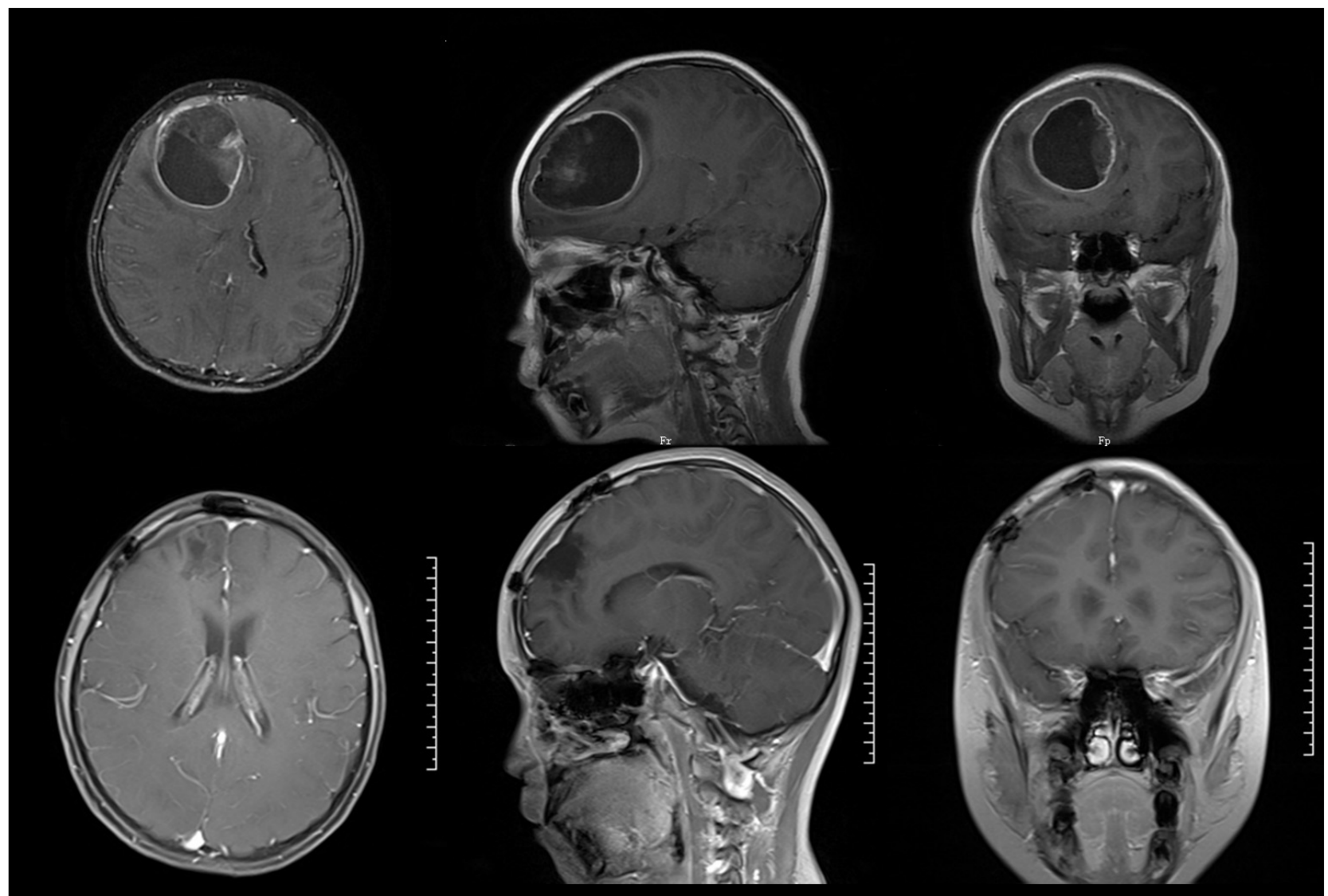

$\mathrm{B}$
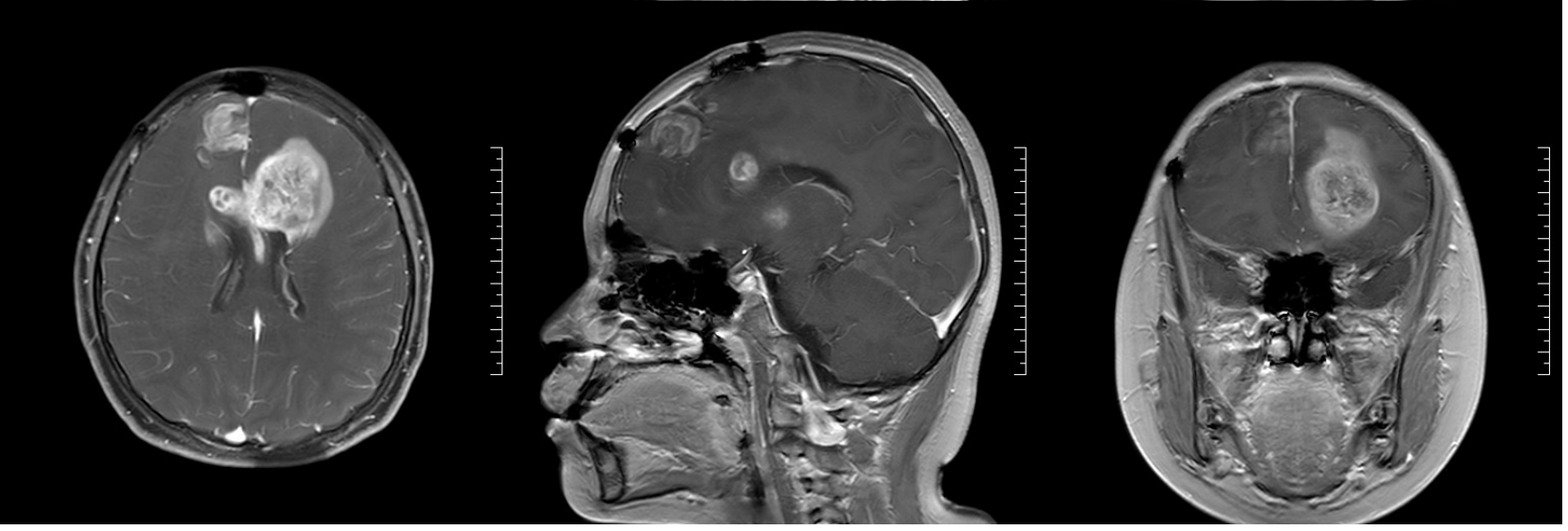

\section{Figure 2}

A special case of tumor recurrence in multiple sites. (A) Preoperative magnetic resonance imaging (MRI) showed tremendous space-occupying lesions with annular enhancement. (B) Three months after the operation, MRI showed that there was no obvious abnormal signal in the operation area, and the tumor was completely removed. (C) Twenty-one months after operation, multiple hyperintense lesions were found in the operation area and contralateral cerebral hemisphere. 

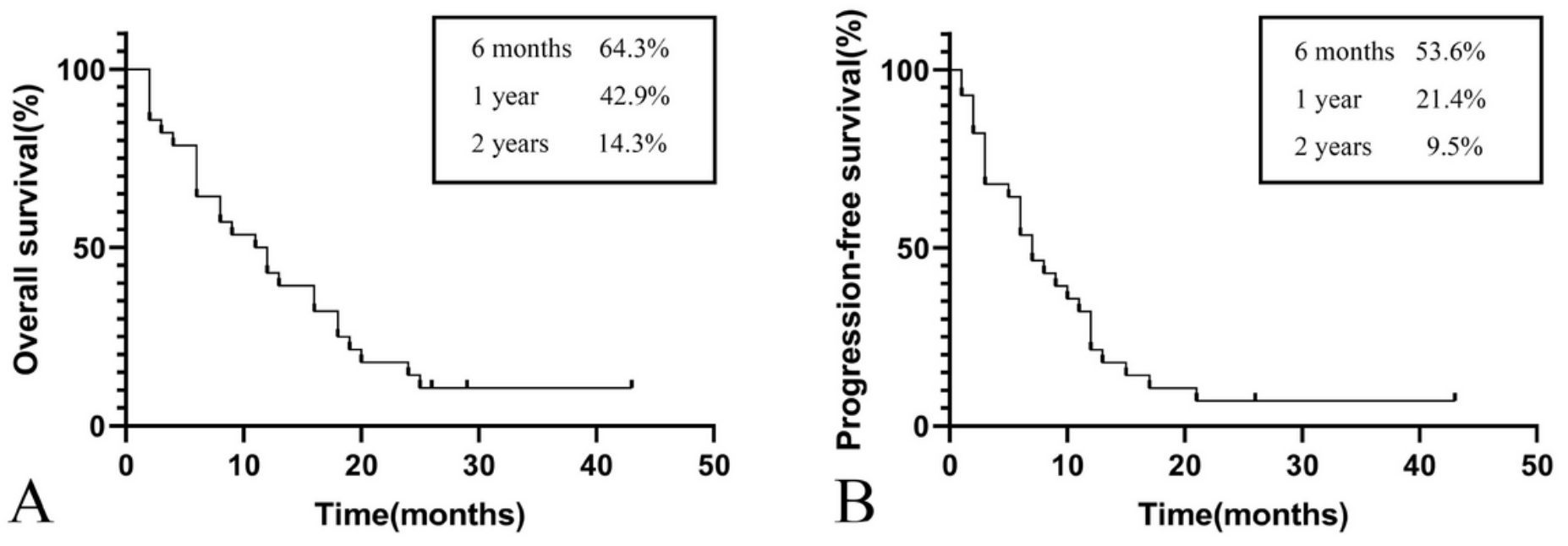

Figure 3

Kaplan-Meier curve. (A) OS Kaplan-Meier curve for 28 patients with psGBM. (B) PFS Kaplan-Meier curve for 28 patients with psGBM. 
A

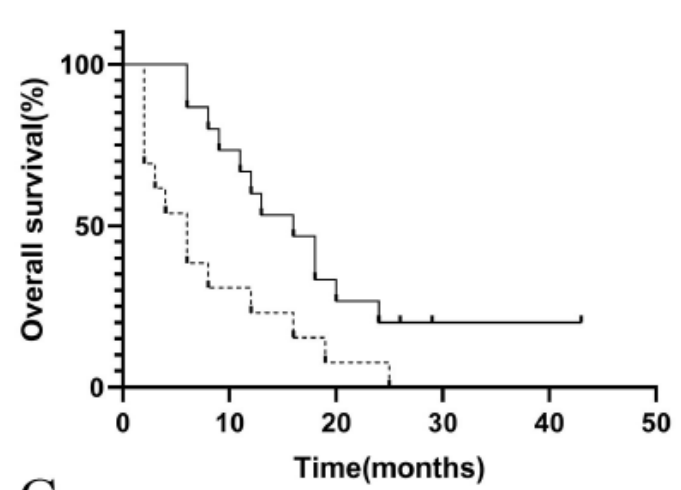

C

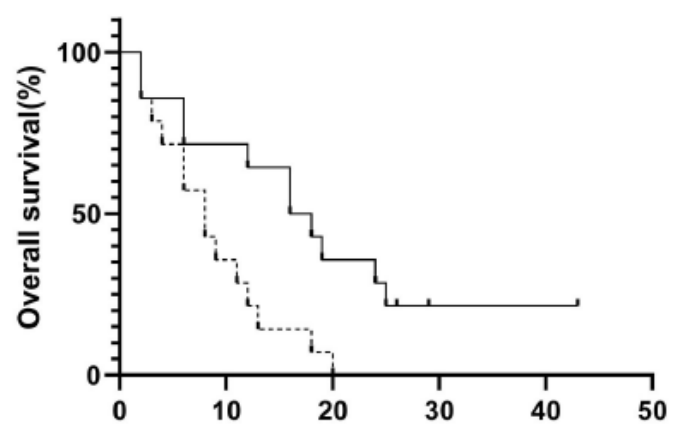

E

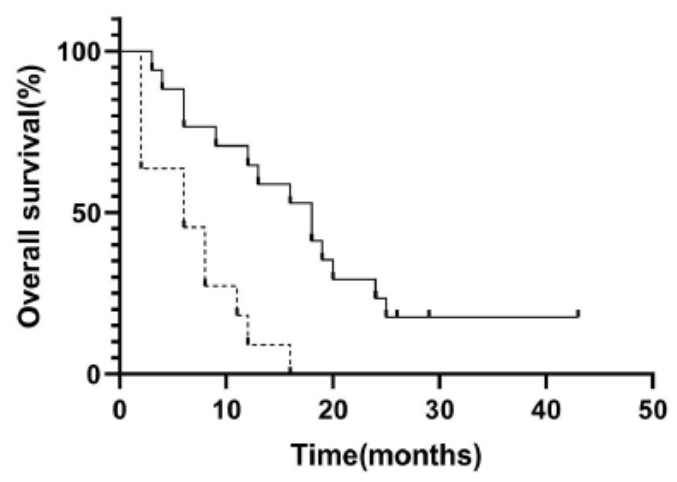

B

- CCRT

.... Non-CCRT

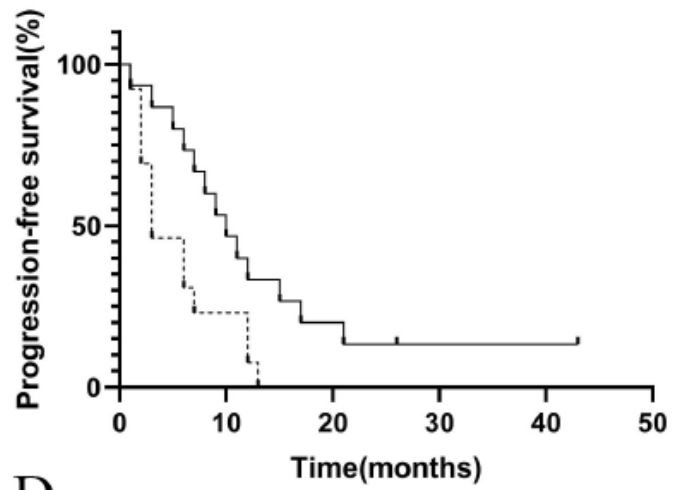

- CCRT

..... Non-CCRT

D

- GTR

.... STR

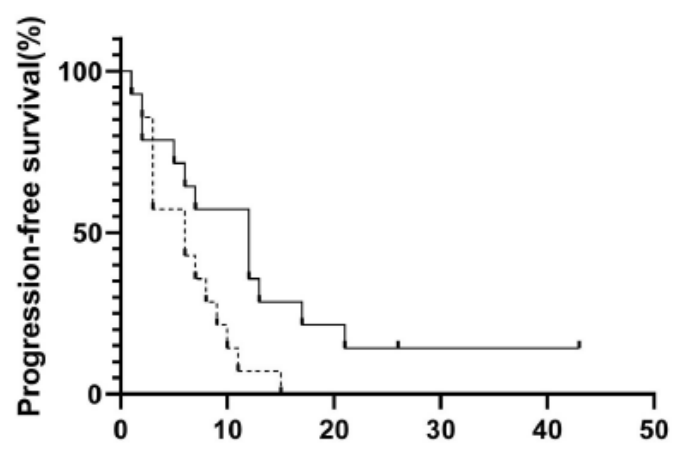

- GTR

.... STR

- $\mathrm{KPS}>70$

.... $\mathrm{KPS} \leqslant 70$

F

Time(months)

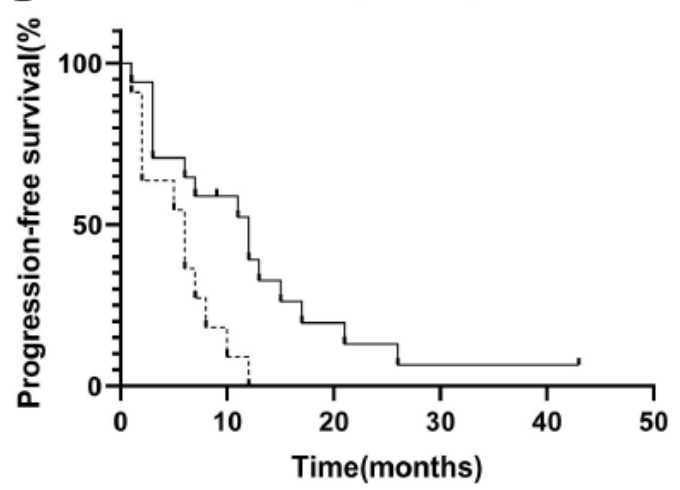

- KPS $>70$

.... $\mathrm{KPS} \leqslant 70$

\section{Figure 4}

Kaplan-Meier curve (A) OS Kaplan-Meier curve for patients got postoperative CCRT or not $(p=0.014)$. (B) PFS Kaplan-Meier curves for patients got postoperative CCRT or not $(p=0.018)$. (C) OS Kaplan-Meier curve for patients got GTR and STR ( $p=0.008)$. (D) PFS Kaplan-Meier curves for patients got GTR and STR $(p=0.022)$. (E) OS Kaplan-Meier curve for patients got preoperative KPS score $\otimes 70$ and $\leq 70$ $(p=0.017)$. (F) PFS Kaplan-Meier curve for patients got preoperative KPS score $\otimes 70$ and $\leq 70(p=0.039)$. 


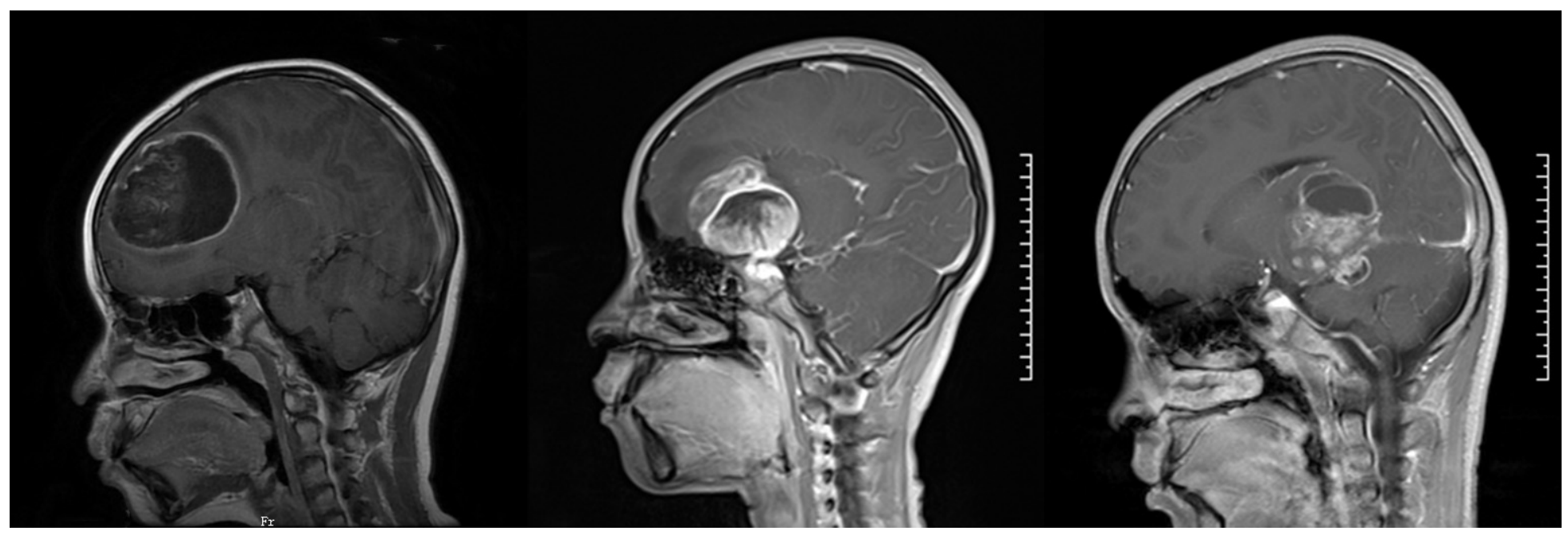

Figure 5

Imaging features of tumors closely related to dura mater. (A) The tumor was located in the superficial part of frontal lobe, and a small part of dura showed enhancement. (B) The tumor is closely related to the dura mater of skull base, and there is no obvious boundary on MRI. (C) The tumor surrounds the tentorium and appears to be connected to it. 